Erminio PIVA*

\title{
Miettiella vespertilio, nuovo genere nuova specie dei Monti Lessini veronesi (Veneto, Italia) \\ (Coleoptera Cholevidae Leptodirinae)
}

\begin{abstract}
Riassunto: In questo lavoro viene descritta Miettiella vespertilio $n$. gen. n. sp. dei Monti Lessini veronesi (Veneto, Italia); per l'insieme dei caratteri risulta appartenere alla "serie filetica di Boldoria". Infine, vengono forniti dati inediti su Monguzziella grottoloi.

Abstract: Miettiella vespertilio, new genus new species from the Lessini Mounts (Verona, Veneto, Italy) (Coleoptera Cholevidae Leptodirinae). In this paper Miettiella vespertilio n. gen. n. sp. from the Lessini Mounts (Verona, Veneto, Italy) is described. Based on the combination of its characters, it belongs to the "phyletic series of Boldoria". Unpublished data on Monguzziella grottoloi are also given.
\end{abstract}

Key words: Coleoptera, Cholevidae, Leptodirinae, Miettiella vespertilio, new genus, new species, Lessini Mounts, Veneto, Italy.

\section{INTRODUZIONE}

Le ricerche biospeleologiche, condotte nell'area veneto-trentina dal Club Speleologico Proteo di Vicenza, hanno fornito notevoli risultati, sia riguardo alle prospezioni in ambienti ipogei profondi (grotte, abissi, voragini), sia in ambiente sotterraneo superficiale (MSS). Scopo di questa nota è la descrizione di una nuova, inattesa specie, rinvenuta in una cavità dei Monti Lessini veronesi e scoperta grazie alla dimenticanza di una trappola a caduta da parte di un anonimo ricercatore. Questo taxon, per l'insieme dei suoi caratteri, risulta ascrivibile ad un genere inedito, più avanti descritto. Inoltre, in questo contributo sono indicate alcune stazioni inedite di Monguzziella grottoloi Vailati, 1993.

Il materiale esaminato è conservato nelle seguenti collezioni: CBI - coll. Dante Bianco, Santorso (Vicenza); CCA - coll. Andrea Carlin, Pergine Valsugana (Trento); CGA - coll. Marco Gabbi, Trento; CGI - coll. Pier Mauro Giachino, Torino; CGR - coll. Mario Grottolo, Brescia; CLA - coll. Leonardo Latella, Verona; CMO - coll. Riccardo Monguzzi, Milano; CPI - coll. Erminio Piva, Vicenza; CVA - coll. Dante Vailati, Brescia; MCVR - Museo Civico di Storia Naturale di Verona; MNAV - Museo Naturalistico Archeologico di Vicenza.

Miettiella $\mathrm{n}$. gen.

SPECIE TIPO: Miettiella vespertilio n. sp.

DiAgnosi. Genere di Cholevidae Leptodirinae di piccola taglia, a facies batiscioide, anoftalmo, attero e de- pigmentato, con scultura elitrale a striole trasversali. Capo retrattile, antenne moderatamente allungate con il primo articolo più breve del secondo. Zampe relativamente brevi e robuste; protarsi maschili pentameri, con il primo articolo molto dilatato. Protibie prive di pettine di spine esterno, meso- e metatibie provviste all'apice di cestelli di spine completi. Carena mesosternale non dentata, con apofisi posteriore libera, prolungata sul metasterno. Edeago tozzo, con parameri sottili, muniti di tre setole apicali; sacco interno con pezzo ad Y basale poco definito, regione mediana munita di fanere complesse, ben sclerificate. Per l'insieme dei caratteri il nuovo genere è attribuibile alla "serie filetica di Boldoria".

DESCRIZIONE. Facies batiscioide; tegumenti depigmentati, ricoperti da fitta pubescenza.

Capo retrattile, robusto, più lungo che largo, con carena occipitale svanita, anoftalmo.

Antenne moderatamente allungate, inserite nel terzo medio del capo, con primo articolo più breve del secondo, il funicolo esile e la clava contratta.

Pronoto trasverso, con la massima larghezza inferiore alla massima larghezza delle elitre.

Elitre brevi e ampie, prive di stria suturale, con pubescenza ordinata su striole trasversali.

Mesosterno munito di carena bene sviluppata, con angolo anteriore arrotondato e privo di dente, con apofisi posteriore libera e prolungata sul metasterno.

Apparato metatergale piccolo, poco sviluppato.

*Erminio Piva, Via Amendola 4, 36100 Vicenza, Italia. E-mail: erminiopiva1@virgilio.it 
Metendosternite conformato a V, con i bracci della furca liberi, sottili e allungati.

Zampe relativamente brevi, ma robuste; protibie prive di pettine lungo il margine esterno, munite all'apice di due speroni polidentati interni, di uno sperone semplice esterno e di una serie di spine lungo il margine superiore. Protarsi maschili pentameri, con primo articolo più largo della protibia; meso- e metatibie provviste all'apice di cestelli di spine completi, di due speroni polidentati interni e di speroni semplici esterni.

Edeago tozzo, parameri sottili muniti di tre setole apicali. Sacco interno con pezzo ad Y basale non ben definito; regione mediana con grandi scleriti di struttura complessa.

Spermateca con il bulbo prossimale allungato e quello distale sferico.

DeRIVATIO NOMINIS. Dedico il nuovo genere al geologo Prof. Paolo Mietto, uno dei fondatori del Club Speleologico Proteo di Vicenza, in segno di ringraziamento della costruttiva e fondamentale collaborazione per oltre un trentennio di attività.

Miettiella vespertilio $\mathrm{n}$. sp. (Figg. 1-9)

Località tiPICA. Veneto, Monti Lessini. Erbezzo (VR); cavità presso Ponte dell'Anguillara, $1020 \mathrm{~m}$ ca. SERIE TIPICA. Holotypus $\widehat{\alpha}$ : Veneto, Monti Lessini. Erbezzo (VR); cavità presso Ponte dell'Anguillara, 1020 m ca., 20.V.2011, E. Piva leg. (CPI). Paratypi: stessa località dell'holotypus: 26.IV.2010, E. Piva leg., 1 ex.

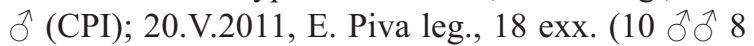
우우) (CBI, CGI, CLA, CPI, CVA, MCVR, MNAV); 12.X.2011, D. Bianco \& E. Piva leg., 3 exx. 우 (CBI, CPI); 15.III.2013, E. Piva leg., 2 exx. (1 $\partial, 1$ q dissezionata) (CPI); 27.IX.2013, E. Piva leg., 1 ex. $\sigma^{\Uparrow}$ (CPI); 20.XI.2014, D. Bianco \& E. Piva leg., 1 ex. $\widehat{\text { ô }}$ (CBI). Lessini Veronesi, Cavità artific. presso Scandole (Erbezzo): vajo d. Anguillara, 19.XI.1998, R. Monguzzi leg., 1 ex. . (CMO).

DERIVATIO NOMINIS. Il nome specifico vespertilio (pipistrello), in apposizione, è riferito alla forma delle sclerificazioni del sacco interno dell'edeago, riproducenti in modo sorprendente la sagoma di questo mammifero. DesCRIZIONE DELl'HOLOTYPus $\hat{\jmath}$. Corpo (Fig. 1) batiscioide, convesso, di colore testaceo, ricoperto da fitta pubescenza dorata, breve e coricata; lunghezza, a capo reclinato, $1.89 \mathrm{~mm}$. Tegumenti lucidi; scultura finissima, a micromaglie poligonali irregolari su capo e pronoto, più marcata e a striole trasversali sulle elitre. Capo anoftalmo, retrattile. Antenne lunghe $1.24 \mathrm{~mm}$ (rapporto lunghezza antenne/lunghezza corpo: 0.66); lunghezza degli antennomeri (in micron): 106-154101-87-90-91-130-67-107-115-188; rapporto lunghezza/larghezza degli stessi: 2.08-3.28-3.26-2.902.73-2.53-1.97-1.56-1.53-1.44-2.38. Organo di Hamann ben sviluppato, occupante i $3 / 5$ della larghezza del settimo antennomero.

Pronoto trasverso, lungo $0.53 \mathrm{~mm}$, largo $0.97 \mathrm{~mm}$ (rapporto lunghezza/larghezza: 0.54), con massima larghezza poco prima della base, che è sinuata; lati arrotondati nel terzo basale, quindi ristretti con debole curvatura in avanti. Angoli posteriori appena acuti. Elitre brevi e larghe, con rapporto lunghezza/larghezza di 1.14 (misurando la lunghezza dal vertice del triangolo scutellare all'apice e la larghezza considerandole assieme); la massima larghezza a circa metà lunghezza. Scultura evidente, costituita da una quarantina

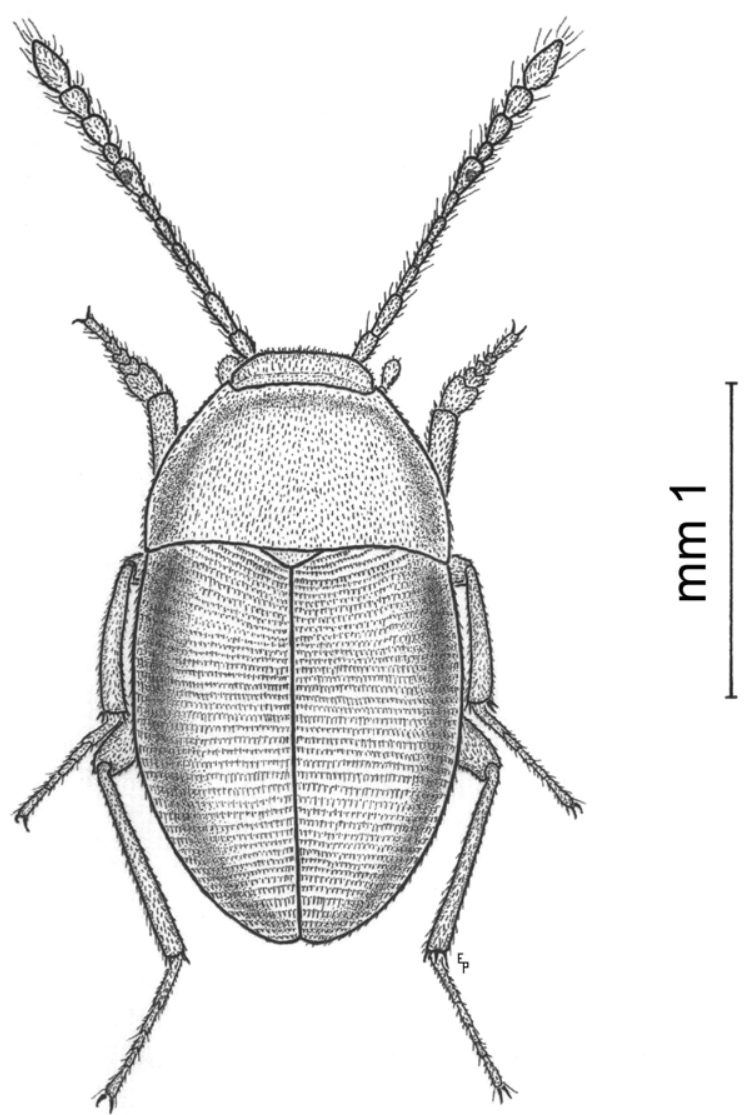

Fig. 1. Miettiella vespertilio $\mathrm{n}$. sp., holotypus $\widehat{\partial}$ : habitus. 
di striole trasversali, con pubescenza regolarmente disposta su queste, le setole lunghe 1-1.5 volte la distanza tra due striole.

Carena mesosternale (Fig. 5) discretamente alta, sottile, con profilo anteriore non prominente e regolar- mente incurvato; angolo anteriore ampiamente arrotondato, profilo ventrale appena concavo in avanti, munito di setole brevi e rade. Apofisi posteriore libera, prolungata per breve tratto sul metasterno.

Zampe relativamente brevi e robuste. Protibie armate
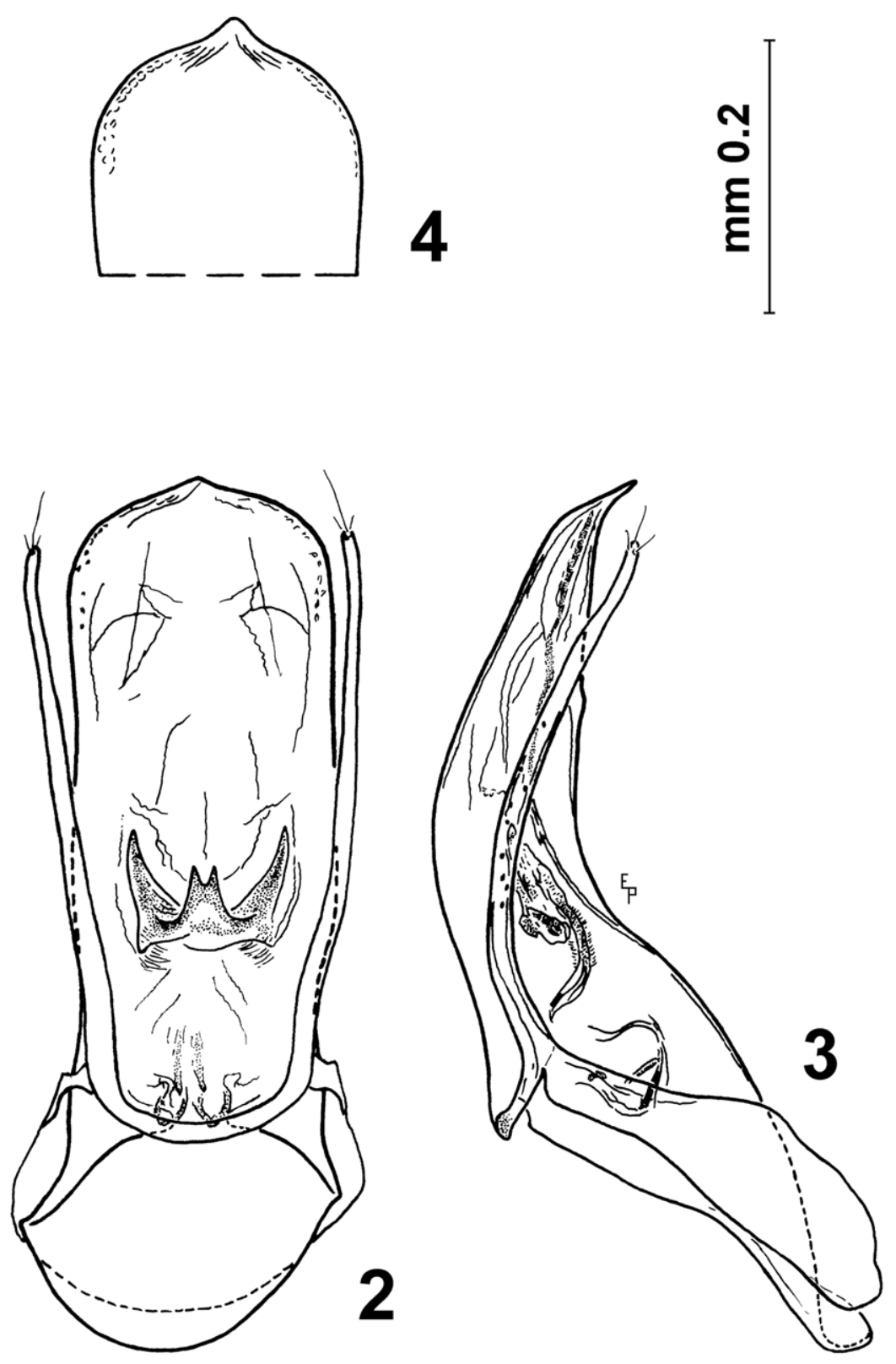

Figg. 2-4. Miettiella vespertilio n. sp., holotypus ${ }^{\lambda}: 2$ - edeago, in visione dorsale; 3 -idem, in visione laterale; 4 -idem, particolare dell'apice. 
all'apice di una serie regolare di spine lungo il margine superiore, di due speroni polidentati interni e di uno sperone semplice esterno. Meso- e metatibie munite di cestello apicale di spine completo, di due speroni polidentati interni e di due speroni semplici esterni. Protarsi pentameri, con primo articolo largo 1.2 volte l'apice della protibia.

Edeago tozzo (Figg. 2-4), lungo $0.64 \mathrm{~mm}$ (rapporto lunghezza edeago/lunghezza corpo: 0.34). Lobo mediano breve e largo, in visione dorsale a lati sinuati e ampiamente arrotondato distalmente, munito di una breve linguetta apicale; in visione laterale (Fig. 3) rigonfio alla base e poco ricurvo nella metà distale, il cui profilo dorsale è rettilineo per un buon tratto. Apice ripiegato ventralmente. Parameri lunghi e sottili, non raggiungenti l'apice del lobo mediano, armati di tre setole. Sacco interno con pezzo ad Y basale non ben definito; regione mediana con una struttura sclerificata riproducente la sagoma di un chirottero.

DESCRIZIONE DEI PARATIPI E VARIABILITÀ. LunghezZa del corpo (a capo reclinato): $1.87 \div 2.00 \mathrm{~mm}$ nei $\widehat{\partial} \widehat{\partial}$, $2.00 \div 2.10 \mathrm{~mm}$ nelle $ᄋ+$. Il rapporto lunghezza antenne/lunghezza corpo è $0.64 \div 0.66$ nei $\widehat{\partial} \widehat{\partial}, 0.52 \div 0.56$ nelle 우우. Le misure (in micron) degli antennomeri di due paratipi, lunghi rispettivamente $1.94 \mathrm{~mm}($ ふ) e

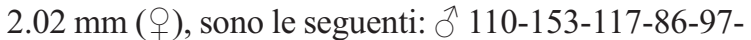
96-131-59-113-114-183; ㅇ 105-140-88-71-77-82110-62-91-87-157. I relativi rapporti lu/la antennomeri sono: के 2.08-3.00-3.25-2.61-2.49-2.18-1.87-1.11$1.71-1.70-2.73$; 우 $1.98-2.80-2.93-2.37-2.48-2.41-$ 1.86-1.51-1.36-1.26-2.21.

Pronoto trasverso, con rapporto lunghezza/larghezza di 0.54-0.59 nei $\widehat{\partial} \widehat{\partial}$, di 0.53-0.58 nelle $\odot \odot$; la massima larghezza presso la base.

Elitre, considerate assieme, con rapporto lunghezza/larghezza di 1.10-1.18 nei $\widehat{\partial} \widehat{\partial}$, di 1.10-1.15 nelle $ᄋ$ 우; striole trasversali in numero variabile.

Carena mesosternale moderatamente alta, con profilo anteriore più o meno incurvato, angolo anteriore arrotondato, quasi sempre ottuso, raramente tendente al retto, profilo ventrale più o meno ampiamente e profondamente incavato; apofisi posteriore spesso prolungata sul metasterno per breve tratto, appena oltrepassante il margine posteriore delle mesoanche, in altri casi più allungata come nell'olotipo.

Paratipi $\widehat{\partial} \hat{\partial}$ conformi all'holotypus per i caratteri genitali (rapporto lunghezza edeago/lunghezza corpo: $0.31 \div 0.34$ ). Il rapporto larghezza del primo tarsomero/larghezza dell'apice della protibia è $1.21 \div 1.28$.
Di un paratipo + , lungo $2.10 \mathrm{~mm}$, sono illustrati l'apparato metatergale del metatergite (Fig. 6), il metendosternite (Fig. 7), l'VIII ventrite (Fig. 8) e la spermateca (Fig. 9); i gonostili, allungati, portano cinque setole: una apicale, tre preapicali e una basale. Negli altri paratipi $q 9$ le setole sono 5 o 6: una apicale, 3 o 4 preapicali, una basale e, raramente, una mediana molto esile.

DisTRIBUZIONE E NOTE ECOLOGICHE. L'unica stazione attualmente nota del nuovo taxon è una cavità artificiale situata a circa 1020 metri nel Vaio dell'Anguilla, tra i paesi veronesi di Erbezzo e Boscochiesanuova; si tratta di un complesso minerario ipogeo relativamente ampio, non più attivo, costituito da alcune gallerie cieche, alcune delle quali soggette a periodici allagamenti.

Tutti gli esemplari sono stati ottenuti da trappole a caduta e nulla conosciamo sulla biologia del nuovo genere. DisCuSSIONE. Dal punto di vista morfologico Miettiella vespertilio presenta analogie sia con alcune specie del genere Boldoria Jeannel, 1924 (facies batiscioide, notevole dilatazione dei protarsi maschili, lobo mediano dell'edeago munito di sclerificazioni ben differenziate, parameri con tre setole in posizione apicale, ottavo ventrite addominale femminile con evidente spiculum mediano) sia con Monguzziella grottoloi (in particolare per la conformazione della carena mesosternale).

Miettiella e Monguzziella risultano essere generi della "serie filetica di Boldoria" presenti entrambi a est del fiume Adige (Fig. 10), dove di norma sono distribuiti elementi "teleomorfi" (sensu Jeannel, 1924), di origine filetica ben diversa. Questa peculiarità zoogeografica, anche se attualmente di difficile comprensione, può avere avuto origine dalle dinamiche conseguenti a diverse ondate di popolamento succedutesi durante l'avvicendarsi delle diverse fasi glaciali pleistoceniche, allorquando le condizioni ambientali e climatiche potevano favorire una dispersione ben maggiore di quella che potrebbe offrire la situazione attuale, caratterizzata da nette barriere fisiche, idrografiche e orografiche. La sorpresa per l'inaspettato ritrovamento di questo nuovo genere, in un sito da parecchi anni indagato da molti biospeleologi, testimonia da un lato l'elevata biodiversità dell'area lessinica, dall'altro la consapevolezza che nessuna regione si può considerare completamente studiata.

Monguzziella grottoloi Vailati, 1993

Monguzziella grottoloi Vailati, 1993: 263; Angelini et al., 1995: 19; Zoia \& Latella, 2005: 179; Giachino \& Vailati, 2005: 282. 

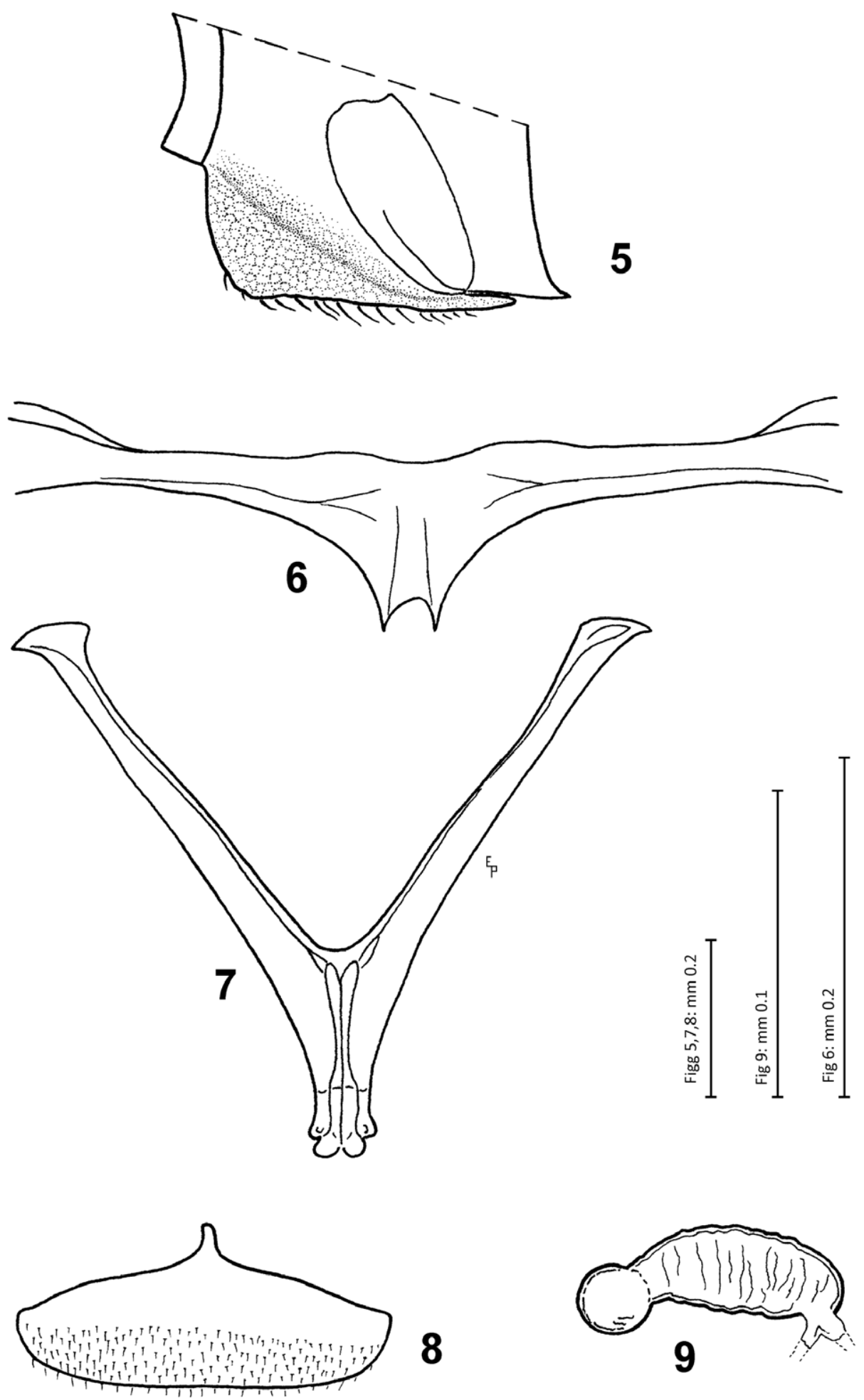

Figg. 5-9. 5 -Miettiella vespertilio n. sp., holotypus ${ }^{\lambda}$ : carena mesosternale; 6 -Miettiella vespertilio $\mathrm{n}$. sp., paratypus ${ }^{+}$(lunghezza corpo: $2.10 \mathrm{~mm}$ ): apparato metatergale; 7 - metendosternite; 8 - VIII ${ }^{\circ}$ ventrite; 9 - spermateca. 
LOCALITÀ TIPICA. Venezia Tridentina, Altipiani di Tonezza-Folgaria e Vigolana. Folgaria (TN); Lont del Laché (419 VT/TN), $1290 \mathrm{~m}$.

DisTRIBUZIONE. Ambienti sotterranei (grotte, MSS) ve- neto-trentini negli Altipiani di Tonezza-Folgaria e Vigolana, nell'Altopiano di Lavarone-Luserna, nel Gruppo del Carega-Piccole Dolomiti-Pasubio (dati inediti) e nei Monti Lessini veronesi.

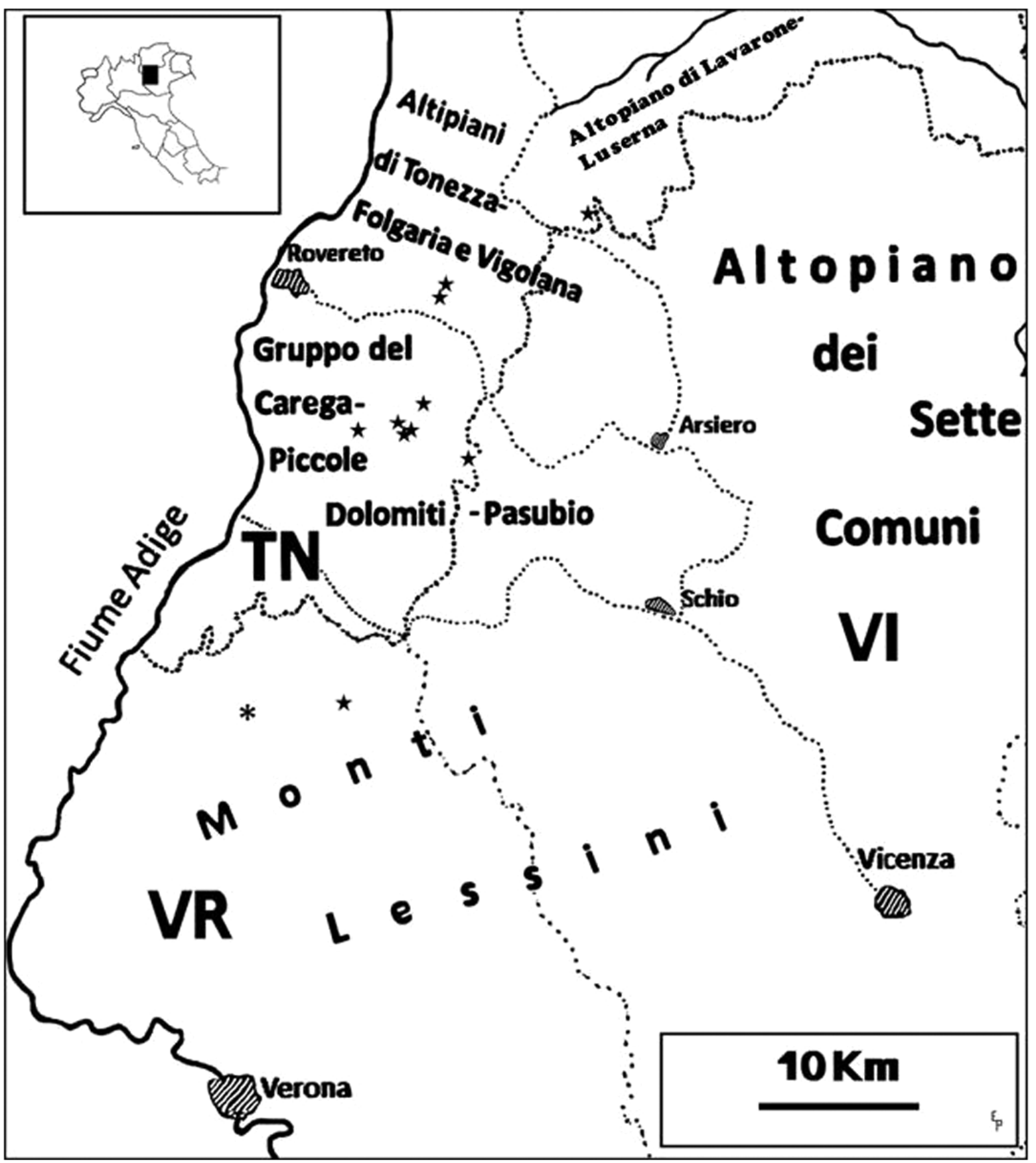

Fig. 10. Cartina di distribuzione. $\star$ Monguzziella grottoloi; * Miettiella vespertilio n. sp. 
Materiale esaminato. Complessivamente 65 exx. (24 수수 41 우 ) : Venezia Tridentina, Altipiani di Tonezza-Folgaria e Vigolana. Folgaria (TN), Lont del Laché (419 VT/TN), 1290 m., 19.IX.1992, M. Grottolo leg., Holotypus ô (CVA); ibidem, 7.XI.2008, M. Gabbi leg., 4 exx. (1 1 ㄱ 3 웅) (CGA, CPI); ibidem,

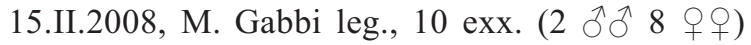
(CBI, CGA, CPI); ibidem, 30.III.2012, D. Bianco \& E. Piva leg., 2 exx. 우우 (CBI, CPI). Venezia Tridentina, Altipiani di Tonezza-Folgaria e Vigolana, Folgaria (TN), Bus del Gobo Onzera (207 VT/TN), 3.V.1992, A. Martinelli leg., 1 paratypus đ̊ (CGR). Venezia Tridentina, Altopiano di Lavarone-Luserna, Lavarone (TN), loc. Oseli, antro alla base di parete rocciosa in faggeta mista, $1230 \mathrm{~m}, 26$.VIII.2007, A. Carlin leg., 12 exx. ( 6 ठぇ 6 ๆ + ) (CBI, CCA, CPI);

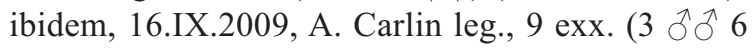
우우) (CCA, CPI); ibidem, 10.VI.2010, A. Carlin leg., 4 exx. (1 $\begin{aligned} & \delta \\ & 3\end{aligned}$ 우 ) (CCA, CPI). Venezia Tridentina, Gruppo del Carega-Piccole Dolomiti-Pasubio, Trambileno (TN), loc. Corno del Pasubio, galleria militare sopra Saletta Comando, $2100 \mathrm{~m}$ ca., 3.VIII.2010, D. Bianco, A. Casa \& E. Piva leg., 1 ex. ㅇ (CPI). Venezia Tridentina, Gruppo del Carega-Piccole DolomitiPasubio, Trambileno (TN), loc. Monte Testo, versante nord-est, in piccola caverna militare, $1850 \mathrm{~m}$, 10.VI.2015, A. Carlin leg., 1 ex. ㅇ (CCA). Venezia Tridentina, Gruppo del Carega-Piccole Dolomiti-Pasubio, Vallarsa (TN), loc. Monte Corno Battisti, Pozzo della Carrucola, 1725 m, 23.VII.2013, D.

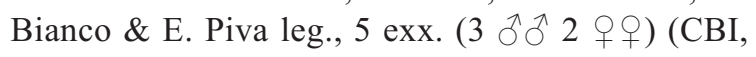
CPI); ibidem, 26.VIII.2014, D. Bianco \& E. Piva leg., 7 exx. (3 $\widehat{\jmath} \widehat{o}^{4}$ 우우) (CBI, CPI). Venezia Tridentina, Gruppo del Carega-Piccole Dolomiti-Pasubio, Vallarsa (TN), loc. Bocchetta di Foxi, $1^{\text {a }}$ galleria, 1725 m, 2.IX.2014, D. Bianco \& E. Piva leg., 1 ex. 우 (CBI). Venezia Tridentina, Gruppo del Carega-Piccole Dolomiti-Pasubio, Vallarsa (TN), loc. Bocchetta di Foxi, $2^{\mathrm{a}}$ galleria, 1725 m, 2.IX.2014, D. Bianco \& E. Piva leg., 1 ex. ㅇ (CPI). Venezia Tridentina, Gruppo del Carega-Piccole Dolomiti-Pasubio, Vallarsa (TN), loc. Bocchetta di Foxi, $3^{\text {a }}$ galleria, 1710 m, 24.VII.2013, D. Bianco \& E. Piva leg., 3 exx. के ô (CBI, CPI); ibidem, 2.IX.2014, D. Bianco \& E. Piva leg., 2 exx. 우우 (CBI, CPI). Veneto, Monti Lessini, Boscochiesanuova (VR), loc. Malga Bagorno, dintorni Grotta dell'Arena, 16.IX.1988, R. Monguzzi leg., 1 paratypus + (CMO).

OsSERVAZIONI. Questa interessante specie è reperibile sui rilievi montuosi veneto-trentini ad est della Valle dell'Adige e, da quanto ho potuto osservare dalle caratteristiche ambientali dei siti di cattura, si raccoglierebbe sporadicamente in particolari microambienti, non necessariamente legati al carsismo profondo, bensì presumibilmente all'orizzonte inferiore del MSS.

Nonostante l'areale relativamente ampio (Fig. 10), la variabilità morfologica dei reperti esaminati, desunta da un esame biometrico, è risultata poco significativa. I dati ottenuti sono i seguenti: lunghezza del corpo (a capo reclinato) $1.74 \div 1.94 \mathrm{~mm}$ (media: $1.87 \mathrm{~mm}$ ) nei

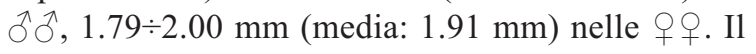
rapporto lunghezza antenne/lunghezza corpo è $0.57 \div 0.62$ (media: 0.58 ) nei $\widehat{\partial}$ ô, $0.51 \div 0.56$ (media: $0.53)$ nelle $q$ ㅇ․ Il rapporto lunghezza/larghezza elitre (misurando la lunghezza dal vertice del triangolo scutellare all'apice elitrale e la larghezza considerandole assieme) è $1.23 \div 1.36$ (media: 1.30 ) nei $\widehat{\text { oे }}$ के, $1.18 \div 1.33$ (media: 1.25) nelle $ㅇ$ ㅇ. $8^{\circ}$ antennomero generalmente trasverso; in otto casi tanto lungo quanto largo e in soli quattro casi più lungo che largo, in una $\mathrm{o}$ in entrambe le antenne. Carena mesosternale talvolta più alta $\mathrm{e}$ con angolo anteriore meno ottuso rispetto a quella rappresentata da Vailati (1993); in alcuni casi il profilo anteriore è moderatamente curvato e in altri quello ventrale è subrettilineo.

La biologia di Monguzziella grottoloi è del tutto ignota e le catture sono avvenute quasi tutte con trappole a caduta (Bianco, Carlin e Monguzzi, comunicazioni personali, nonché mie osservazioni). Su dieci stazioni di rinvenimento ben sei riguardano microambienti costituiti da frane di materiale clastico in gallerie e ricoveri militari (in particolare sul M. Pasubio), tre stazioni riguardano cavità naturali o artificiali e una l'ambiente sotterraneo superficiale (presso Malga Bagorno). Si potrebbe supporre che l'ambiente di vita ottimale di questi insetti sia alla base del MSS, a contatto della roccia madre (l'interfaccia tra gli orizzonti $\mathrm{C} 1 \mathrm{e}$ C2 sensu Juberthie et al., 1980) e che la presenza in grotta sia del tutto occasionale (per temporaneo abbandono del normale habitat o per esserne a questo contiguo); eccezionale e dovuto probabilmente alle medesime cause l'unico ritrovamento in ambiente sotterraneo superficiale.

\section{RINGRAZIAMENTI}

Sono grato a tutti coloro che in varia misura hanno reso possibile questo lavoro: per primo all'anonimo raccoglitore che, con la sua dimenticanza, mi ha 
permesso di scoprire la nuova entità; a Leonardo Latella (Museo Civico di Storia Naturale di Verona) e al personale del Parco Naturale Regionale della Lessinia, per la concessione dei permessi di raccolta di microfauna nell'area protetta; ad Andrea Carlin (Pergine Valsugana, Trento), Marco Gabbi (Trento) e Riccardo
Monguzzi (Milano), per avermi consentito di esaminare i loro reperti.

Ringrazio, inoltre, Dante Bianco (Santorso, Vicenza), attivo e abile compagno di ricerche, nonché Roberto Poggi (Genova), Pier Mauro Giachino (Torino) e Dante Vailati (Brescia), per gli utili consigli.

BIBLIOGRAFIA

Angelini F., Audisio P., Castellini G., Poggi R., Vailati D., Zanetti A., Zoia S., 1995 - Coleoptera Polyphaga II (Staphylinoidea escl. Staphylinidae), 39 pp. In: A. Minelli, S. Ruffo, S. La Posta (eds). Checklist delle specie della fauna italiana, 47, Calderini, Bologna.

Giachino P.M., VAILATi D., 2005 - I Cholevidae delle Alpi e Prealpi italiane: inventario, analisi faunistica e origine del popolamento nel settore compreso fra i corsi dei fiumi Ticino e Tagliamento (Coleoptera). Biogeographia, Lavori della Società italiana di Biogeografia, (nuova serie), 26: 229-378.

Jeannel R., 1924 - Biospeologica L. Monographie des Bathysciinae. Archives de Zoologie expérimentale et générale, 63 : 1-436.

Juberthie C., Delay B., Bouillon M., 1980 - Extension du milieu souterrain en zone non calcaire: description d'un nouveau milieu et de son peuplement par les Coléoptères troglobies. Mémoires de Biospéologie, 7: 19-52.

Sbordoni V., Rampini M., Cobolli Sbordoni M., 1982 - Coleotteri Catopidi cavernicoli italiani. Lavori della Società italiana di Biogeografia, (nuova serie), 7 (1978): 253-336.

VAILATI D., 1988 - Studi sui Bathysciinae delle Prealpi centro-occidentali. Revisione sistematica, ecologia, biogeografia della "serie filetica di Boldoria" (Coleoptera Catopidae). Monografie di "Natura Bresciana", 11: 1-331.

VAILATI D., 1993 - Monguzziella grottoloi nuovo genere nuova specie delle Prealpi Venete (Coleoptera Cholevidae Leptodirinae). "Natura Bresciana” Annali del Museo Civico di Scienze Naturali, Brescia, 28 (1992): 261-278.

Zoia S., Latella L., 2005 - Insecta Coleoptera Cholevidae e Platypsyllidae. In: Ruffo S., Stoch F. (eds.). Checklist e distribuzione della fauna italiana. Memorie del Museo Civico di Storia Naturale di Verona, 2a serie, Sezione Scienze della Vita, 16: 177-180. 